\section{CONTENTS OF NUMBER 7}

Transformation of 12-deoxycardenolids with Streptomyces purpurascens

Cyanide-resistant respiration in a respiration-defieient mutant of Saccharomyces cerevisiae

Arthrobacter globiformis - a bacterium capable to lyse viable yeast cells

Regulation of PEP-carboxylase from the facultative methylotrophic Acetobacter sp. MB 58

A new major outer membrane protein in derivatives of Escherichia coli carrying the virulence plasmid ColV-K94

Polysaceharide structures of cell wall preparations from the food protein yeast Candida spec. H

Characterization of a $\mathrm{CaCl}_{2}$-dependent transfection system from Escherichia coli and T3 bacteriophage DNA

Regulation of protein biosynthesis in outgrowing spores of Bacillus subtilis

Short Note

The temperature profile of growth, death and yield of the starch-converting yeast Lipomyces kononenkoae

Book Reviews
K. Albrecht, G. Máté and T.LÁNg

J. D. Arrabaça and M. C. Loureiro Dias 437

D. BLECHSCHMidT, C. GoHr and R. TRÖGER 443

N. LOFFHAGEN and W. BABEL

JANE C. Moores and R. J. Row. BURY

J. NÜSKe, H. D. Grimmecke and G. REUTER

H. Stompe, S. Michel, W. ManN and G. RICHTER'

G. WACHLIN and M. HECKER 495

I. SPENCER-Martins and N. van UDEN

\section{INHALTSVERZEICHNIS HEFT 7}

Transformation von 12-Desoxycardenoliden mit Streptomyces purpurascens

K. Albrecht, G. Máté und

T. LÁNG

Cyanid-resistente Atmung in einer atmungsdefekten Mutante von Saccharomyces cerevisiae

J. D. Arrabaga und M. C. LoureiRo Dias

Arthrobacter globiformis - ein neues hefelytisches Bakterium

D. BLECHSCHMIDT, C. GOHR und

R. TRÖGER

N. LOFFHAGEN und W. BABEL methylotrophen Acetobacter sp. MB 58

Ein neues Protein bei Escherichia coli-Abkömmlingen mit dem Virulenzplasmid ColV-K94

JANE C. MOORES und R. J. RowBURY

Polysaccharid-Strukturen von Zellwand-Präparaten aus der Futtereiweiß-Hefe Candida spec. $\mathrm{H}$

J. NüsKe, H. D. Grimmeoke und G. REUTER

Charakterisierung eines $\mathrm{CaCl}_{2}$-abhängigen Transfektionssystems von Escherichia coli und T3Phagen-DNS

Aktivierung von Proteinbiosynthesen in auswachsenden Sporen von Bacillus subtilis

H. Stompe, S. Michel, W. ManN und G. RICHTER

G. WACHLIN und M. HECKER

\section{Kurze Originalmitteilung}

Temperaturprofil von Wachstum, Tod und Ertrag der Stärke-umwandelnden Hefe Lipomyces kononenkoae

I. SPENCER-Martins und N.vaN UDEN

Buchbesprechungen 


\section{ZEITSCHRIFT} FƯR

ALLGEMEINE

MIKRO-

-BIOLOGIE

AN INTERNATIONAL JOURNAL ON MORPHOLOGY, PHYSIOLOGY, GENETICS, AND ECOLOGY OF MICROORGANISMS
HERAUSGEGEBEN VON

F. Egami, Tokio

G. F. Gause, Moskau

O. Hoffmann-Ostenhof, Wien

A. A. Imšeneckii, Moskau

R. W. Kaplan, Frankfurt/M:

F. Mach, Greifswald

I. Málek, Prag

C. Weibull, Lund unter der Chefredaktion von

W. Schwartz, Braunschweig und

U. Taubeneck, Jena

UNTER MITARBEIT VON

J. H. Becking, Wageningen

H. Böhme, Gatersleben

M. Girbardt, Jena

S. I. Kusnecov, Moskau

O. Nečas, Brno

C. H. Oppenheimer,

Port Aransas

N. Pfennig, Göttingen

I. L. Rabotnova, Moskau

A. Schwartz, Wolfenbüttel

HEFT $7 \cdot 1982 \cdot$ BAND 22

REDAKTION

U. May, Jena 
Die Zeitschrift für Allgemeine Mikrobiologie soll dazu beitragen, Forschung und internationale Zusammenarbeit auf dem Gebiet der Mikrobiologie zu fördern. Es werden Mausnkripte aus allen Gebieten der allgemeinen Mikrobiologie veröffentlicht. Arbeiten über Themen aus der medizinischen, landwirtschaftlichen, technischen Mikrobiologie und aus der Taxonomie der Mikroorganismen werden ebenfalls aufgenommen, wenn sie Fragen von allgemeinem Interesse bebehandeln.

Zur Veröffentlichung werden angenommen:

Originalmanuskripte, die in anderen Zeitschriften noch nicht veröffentlicht worden sind und in gleicher Form auch nicht in anderen Zeitschriften erscheinen werden. Der Umfang soll höchstens $l^{1} / 2$ Druckbogen (24 Druckseiten) betragen. Bei umfangreicheren Manuskripten müssen besondere Vereinbarungen mit der Schriftleitung und dem Verlag getroffen werden. Kurze Originalmitteilungen über wesentliche, neue Forschungsergebnisse. Umfang im allgemeinen höchstens 3 Druckseiten. Kurze Originalmitteilungen werden beschleunigt veröffentlicht.

Kritische Sammelberichte und Buchbesprechungen nach Vereinbarung mit der Schriftleitung.

Bezugsmöglichkeiten der Zeitschrift für Allgemeine Mikrobiologie:

Bestellungen sind zu richten

- in der DDR an den Postzeitungsvertrieb, an eine Buchhandlung oder an den Akademie-Verlag, DDR-1086 Berlin, Leipziger Straße 3-4

- im sozialistischen Ausland an eine Buchhandlung für fremdsprachige Literaur oder an den zuständigen Postzeitungsvertrieb

- in der BRD und Berlin(West) an eine Buchhandlung oder an die Auslieferungsstelle KUNST UND WISSEN, Erich Bieber, D-7000 Stuttgart 1, Wilhelmstraße 4-6

- in den übrigen westeuroäpischen Ländern an eine Buchhandlung oder an die Auslieferungsstelle KUNST UND WISSEN, Erich Bieber GmbH, CH-8008 Zürich, Dufourstraße 51

- im übrigen Ausland an den Internationalen Buch- und Zeitschriftenhandel; den Buchexport, Volkseigener Außenhandelsbetrieb der Deutschen Demokratischen Republik, DDR-7010 Leipzig, Postfach 160, oder an den Akademie-Verlag, DDR-1086 Berlin, Leipziger Straße 3-4.

Zeitschrift für Allgemeine Mikrobiologie

Herausgeber: Im Auftrag des Verlages von einem internationalen Wissenschaftlerkollektiv herausgegeben.

Verlag:Akademie-Verlag,DDR-1086Berlin, Leipziger Straße 3-4; Fernruf : 2236229 oder 2236221

Telex-Nr. : 114420; Bank: Staatsbank der DDR, Berlin, Kto-Nr.: 6836-26-20712.

Chefredaktion: Prof. Dr. Udo TAUBENECK.

Prof. Dr. Wilhelm Schwartz

Anschrift der Redaktion: Zentralinstitut für Mikrobiologie und experimentelle Therapie der Akademie der Wissenschaften, DDR-6900 Jena, Beutenbergstr. 11; Fernruf: Jena 885614; TelexNr. : 058621 .

Veröffentlicht unter der Lizenznummer 1306 des Presseamtes beim Vorsitzenden des Ministerrates der Deutschen Demokratischen Republik.

Gesamtherstellung: VEB Druckerei ,Thomas Müntzer“, DDR-5820 Bad Langensalza.

Erscheinungsweise: Die Zeitschrift für Allgemeine Mikrobiologie erscheint jährlich in einem Band mit 10 Heften. Bezugspreise je Band $220,-\mathrm{M}$ zuzüglich Versandspesen (Preis für die DDR $200,-\mathrm{M}$ ). Preis je Heft $22,-\mathrm{M}$ (Preis für die DDR 20,-M).

Urheberrecht: Alle Rechte vorbehalten, insbesondere die der Übersetzung. Kein Teil dieser Zeitschrift darf in irgendeiner Form - durch Photokopie, Mikrofilm oder irgendein anderes Verfahren - ohne schriftliche Genehmigung des Verlages reproduziert werden. - All rights reserved (including those of translations into foreign languages). No part of this issue may be reproduced in any form, by photoprint, microfilms or any other means, without written permission from the publishers.

Erscheinungstermin: Oktober 82

Bestellnummer dieses Heftes 1070/22/7

(C) 1982 by Akademie-Verlag Berlin. Printed in the German Democratic Republic. AN (EDV) 75218 